\title{
Greetings and Foreword
}

\author{
Dear Colleagues,
}

This special issue of NOLTA journal is based on the NOLTA 2013 conference in Santa Fe, New Mexico. At this conference, NOLTA was looking for the new directions to expand, at the same time building on existing strengths in theory and applications of the nonlinear phenomena. This conference yielded a wonderful opportunity for researchers to catch up in the recent development in research, and establish new collaborations, especially on the international level.

We would like to acknowledge technical program co-chairs Prof. Juan Restrepo (Arizona) and Dr. Yuzuru Sato (Hokkaido), as well as Dr. Kohei Yamasue (Tohoku), for their role in shaping the scientific direction of the conference. The scientific program, encompassing a wide variety of topics in nonlinear phenomena with over 160 submitted abstracts and multiple special sessions, was expertly handled by the special session co-chairs Profs. Jeffrey Moehlis (UCSB) and Masayuki Sato (Kanazawa). General secretary Dr. Yoshihiko Susuki (Kyoto) has done incredible work in juggling multiple organizational tasks so all conference deadlines could be met. Financial questions (sometimes of a challenging nature) were handled beautifully by finance chair Prof. Yuichi Tanji (Kagawa) and finance secretary Dr. Ken'ichi Fujimoto. Publication chair Prof. Hiroyuki Torikai (Kyoto Sangyo) and co-secretaries Drs. Masayuki Kimura (Shiga Prefecture) and Yuichi Yokoi (Nagasaki) have worked very hard to keep the submission and publication of abstracts on time and in the correct formats provided. We also acknowledge Dr. Kimura's artistic efforts in designing various NOLTA 2013 logos. The contribution of publicity chair Dr. Haruna Matsushita (Kagawa) was essential in advertising and popularizing the conference. Finally, we would like to acknowledge the hard work of all other participants of the NOLTA 2013 organizing committee, without whose hard work and dedication the smooth organization of this conference would have been impossible.

For this special issue, we would like to acknowledge the work of Associate Guest Editors: Drs. Ken'ichi Fujimoto, Yoshito Hirata, Visarath In, Masayuki Kimura, Keiji Konishi, Takuji Kousaka, Masayuki Sato, Ryo Takahash, Isao Tokuda, Hiroyuki Torika, Kazuyuki Yashimur. Their work in editing the manuscripts is the key to what we hope will be a highly read and valued issue of NOLTA journa.

We hope to see you personally at the next NOLTA conference, and read your future submissions in NOLTA journal.

With best regard,

Dr. Yuzuru Sato (Hokkaido University), Prof. Vakhtang Putkaradze (University of Alberta)

Guest Editors 